\title{
On the physical origin of the nozzle characteristic and its connection with the double-arcing phenomenon in a cutting torch
}

\author{
L. Prevosto, ${ }^{1}$ H. Kelly, ${ }^{2, a)}$ and B. Mancinelli ${ }^{1}$ \\ ${ }_{1}^{1}$ Universidad Tecnológica Nacional, Regional Venado Tuerto, Las Heras 644, Venado Tuerto (2600), Pcia. \\ Santa Fe, Argentina \\ ${ }^{2}$ Departamento de Física, Instituto de Física del Plasma (CONICET), Facultad de Ciencias Exactas y \\ Naturales (UBA), Ciudad Universitaria, Pab. I, Buenos Aires 1428, Argentina
}

(Received 14 May 2008; accepted 31 October 2008; published online 9 January 2009)

\begin{abstract}
The nozzle current-voltage characteristic for a cutting arc is presented in this work. The measurements are reported using a high energy density cutting arc torch with a nozzle bore radius of $0.5 \mathrm{~mm}$. The arc current was fixed at $30 \mathrm{~A}$ while the plenum pressure and the oxygen gas mass flow rate were varied in the range of $0.55-0.65 \mathrm{MPa}$ and $0.32-0.54 \mathrm{~g} \mathrm{~s}^{-1}$, respectively. The results show a very low electron density and the lack of electron attachment at the plasma boundary layer. No ion saturation current was found. For the smallest mass flow rate value gas breakdown was found for a biasing nozzle potential close to that of the cathode, but no evidence of such breakdown was found for the larger mass flow rate values. Using an expression for the ion speed at the entry of the collisional sheath formed between the nonequilibrium plasma and the negatively biased nozzle wall together with a generalized Saha equation coupled to the ion branch of the characteristic, the radial profile of the electron temperature, the spatial distribution of the plasma density at the plasma boundary, and the sheath thickness were obtained. In particular, the obtained thickness value at the breakdown condition was in good agreement with that obtained from the oxygen Paschen's curve. An electron temperature of about 4700-5700 K and a corresponding plasma density of the order of $10^{19}-10^{20} \mathrm{~m}^{-3}$ were found close to the nozzle wall. A physical interpretation on the origin of the double-arcing phenomenon is presented, that explains why the double-arcing (that it is established when the sheath breaks down) appears at low values of the gas mass flow. (C) 2009 American Institute of Physics. [DOI: 10.1063/1.3041636]
\end{abstract}

\section{INTRODUCTION}

Plasma cutting is a process of metal cutting at atmospheric pressure by an arc plasma jet, where a transferred arc is generated between a cathode and a workpiece (the metal to be cut) acting as the anode. A high-quality cut requires a narrow, hot and high-velocity plasma jet, i.e., a high energy density arc jet. To this end, a new generation of cutting torches, the so-called high energy density torch has been developed. These torches are characterized by an arc current intensity in the range of 30-100 A, flat cathodes, oxygen as the plasma gas, very small nozzle diameters $(\approx 1 \mathrm{~mm})$ and by the generation of an underexpanded supersonic arc jet with a shock wave close to the nozzle exit. ${ }^{1-6}$

The most explored region of plasma in the arc cutting system is the arc column located between the nozzle exit and the workpiece. The experimental data are somewhat scarce, and they are obtained usually by noninvasive spectroscopic techniques. $^{2-5}$ Recently, also Langmuir probes have been used for plasma diagnostics in this kind of arcs. ${ }^{7,8}$ However, due to the smallness of the nozzle bore, and the hostile conditions prevailing inside such arcs, access to experimental information about the plasma state in the nozzle region is out of reach to most plasma diagnostics, and numerical simula-

${ }^{a)}$ Electronic mail: kelly@tinfip.lfp.uba.ar. tions are the only mean to obtain information on the plasma structure. In fact, no experimental data of plasma parameters inside the nozzle is available. ${ }^{9}$

Most of the developed numerical simulations to study the plasma structure are based on plasma local thermodynamic equilibrium (LTE) ${ }^{3,4,6}$ However, substantial deviations from LTE should occur in the arc boundary inside the torch, where the electron density $n_{e}$ is presumably much lower than $10^{23} \mathrm{~m}^{-3}$ (the lower limit for the existence of such equilibrium in a homogeneous plasma, according to Griem's criterion $^{10}$ ); and where very high temperature gradients may be present over the last few electron Debye lengths from the nozzle wall. Recently, Ghorui et al. ${ }^{11}$ presented a nonequilibrium modeling of a 200 A oxygen-plasma cutting torch. It was shown that the electron temperature remained high near the nozzle wall and hence well decoupled from the heavy particle temperature. For instance, an electron temperature of about $12000 \mathrm{~K}$ was reported in the arc boundary at the nozzle exit (a value much higher than the heavy particle temperature of about $1000 \mathrm{~K}$ close to the inner nozzle wall temperature).

In this work measurements of the nozzle current-voltage characteristic are reported for a high energy density oxygen cutting torch. No saturation ion current was found. Note that the metallic nozzle is itself the Langmuir probe, so the plasma flow is not perturbed by the probe as a solid body and the thermal effects of the probe become a boundary condition to the arc. Current-voltage characteristics corresponding 
to large-sized plasma walls in high pressure arcs were obtained only for wall-stabilized arcs with or without imposed gas flow. ${ }^{12,13}$

As it is well known, there is no comprehensive theory for interpreting the electron branch in highly collisional plasmas, hence only the ion branch is analyzed in this work. The influence of the collisions on the physical structure of the plasma-sheath transition has been considered under the typical assumption of constant ion mean free path. Within this assumption and using an approximate analytical solution for the sheath thickness, the value of the ion flux to the wall was related to the nozzle voltage and the electron temperature and density at the plasma boundary. To describe this plasma composition an appropriate nonequilibrium two-temperature statistical model was used. Since the sheath thickness depends on the nozzle voltage value and the collected current depends on the unperturbed plasma quantities values at the sheath edge, the plasma is "probed" at different depths, and hence an appropriate way to explain the observed behavior of the ion branch characteristic is to assume relevant radial variations of the plasma quantities. Thus, fitting the ion branch of the characteristic, the radial profile of the electron temperature and density at the plasma boundary can be obtained.

An estimation of the photoelectron current from the nozzle inner surface due to the UV electromagnetic radiation, and its contribution to the characteristic curve is presented and discussed.

One of the main drawbacks that puts a limit to increasing capabilities of plasma arc cutting process is the presence of a double arcing (where the arc is split into two: one connecting the cathode and the nozzle, the other connecting the nozzle and work piece). A physical interpretation on the origin of this phenomenon is presented, that explains why the double arcing (that it is established when the sheath breaks down) appears at low values of the gas mass flow.

\section{EXPERIMENT}

The high energy density cutting torch used in this study consisted of a cathode centered above an orifice in a converging-straight copper nozzle without liquid cooling. The cathode was made of copper ( $7 \mathrm{~mm}$ in diameter) with a hafnium tip (1.5 $\mathrm{mm}$ in diameter) inserted at the cathode center. A flow of oxygen gas cooled the cathode and the nozzle and was also employed as the plasma gas. The gas passed through a swirl ring to provide arc stability. The nozzle consisted in a converging-straight bore (with a bore radius $R_{N}=0.5 \mathrm{~mm}$ and a length $L_{N}=4.5 \mathrm{~mm}$ ) in a copper holder surrounding the cathode (with a separation of $0.5 \mathrm{~mm}$ between the holder and the cathode surface). To avoid plasma contamination by metal vapors from the anode, a rotating steel disk with $200 \mathrm{~mm}$ in diameter and $15 \mathrm{~mm}$ thickness was used as the anode. ${ }^{1,4}$ In this study, the disk upper surface was located at $5 \mathrm{~mm}$ from the nozzle exit. The arc was transferred to the edge of the disk, and the rotating frequency of the disk was equal to $29.5 \mathrm{~Hz}$. At this velocity, a well-stabilized arc column was obtained, and the lateral surface of the anode disk was completely not melted. Thus,

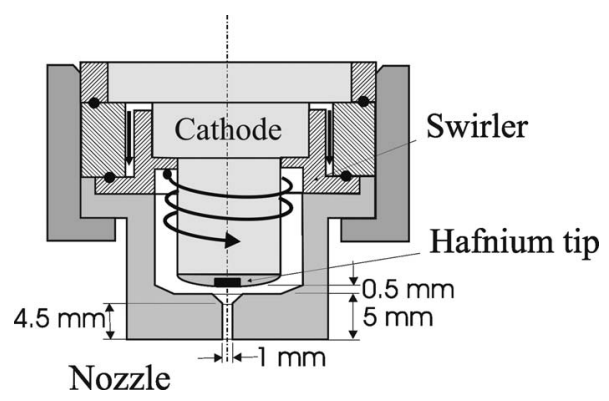

FIG. 1. Scheme of the arc torch indicating several geometric dimensions.

practically no metal vapors from the anode were present in the arc. A scheme of the torch indicating several geometric dimensions is presented in Fig. 1.

By performing a small orifice (1 $\mathrm{mm}$ in diameter) on the lateral of the cathode surface the pressure in the plenum chamber $\left(p_{\mathrm{ch}}\right)$ was measured by connecting a pressure meter at the upper head of the cathode. The gas mass flow $(\dot{m})$ injected in the torch was also registered. In this experiment, the arc current was fixed at $30 \mathrm{~A}$, while $p_{\text {ch }}$ and $\dot{m}$ were varied in the range, $0.55-0.65 \mathrm{MPa}$ and $0.32-0.54 \mathrm{~g} \mathrm{~s}^{-1}$, respectively.

To obtain the current-voltage nozzle characteristic, it was necessary to bias the nozzle (that under normal arc operation remains floating). The nozzle biasing circuit is shown in Fig. 2. Different nozzle bias voltages $\phi_{N}$ were obtained using a resistive voltage divider $(50 \mathrm{k} \Omega$ rheostat), and were registered (with respect to the grounded anode) by using a high-impedance $(10 \mathrm{M} \Omega$ ) voltage meter. The nozzle current $i$ was calculated from the voltage drop $\phi_{0}$ through a small resistance $R_{0}$. In practice, the minimum $\phi_{N}$ value that can be reached with this method was very close to the cathode voltage $(=-165 \pm 5 \mathrm{~V})$. Alternatively, the nozzle was disconnected to perform nozzle floating voltage measurements. To build the ion branch of the $i-\phi_{N}$ curve, the rheostat terminal $a$ was connected to the cathode terminal $b$; while it was connected to the anode terminal $c$ to obtain the electron branch.

A set of sweeping electrostatic probes previously developed $^{7}$ was here employed to determine the plasma floating potential at the nozzle exit for the quoted investigated values of $p_{c h}$ and $\dot{m}$.

\section{RESULTS AND INTERPRETATION}

In Fig. 3(a), a typical $i-\phi_{N}$ nozzle characteristic curve is shown for $\dot{m}=0.54 \mathrm{~g} \mathrm{~s}^{-1}$ and $p_{\mathrm{ch}}=0.65 \mathrm{MPa}$, with the elec-

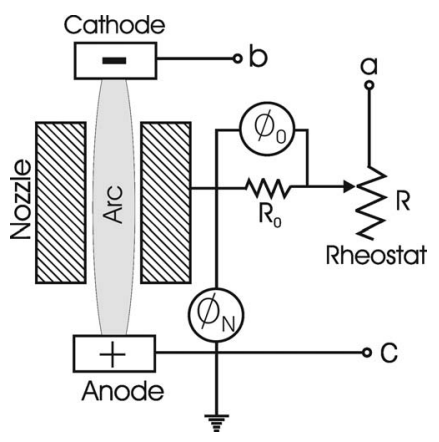

FIG. 2. Scheme of the nozzle biasing circuit. 

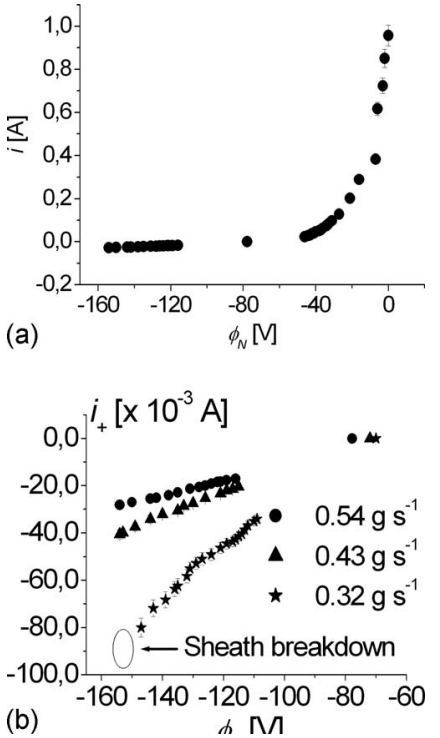

FIG. 3. (a) Nozzle current-voltage characteristic (with the electron current assumed as positive) for the largest gas mass flow used $\dot{m}=0.54 \mathrm{~g} \mathrm{~s}^{-1}$. (b) Detail of the ion branches for several gas mass flow values with an enlarged current scale. Note that for the lowest gas mass flow value the sheath breaks down at a nozzle biasing voltage $\phi_{N}=-155 \pm 5 \mathrm{~V}$.

tron current considered as positive. In Fig. 3(b) a detail of the ion branch with an enlarged current scale is shown. For comparison purposes the ion branch corresponding to other $\dot{m}$ values has been included in Fig. 3(b). The intersection points of the ion branches with the zero-current line (i.e., the nozzle floating voltage values) are clearly identified. For the smallest $\dot{m}$ value gas breakdown was found for $\phi_{N}=-155 \pm 5 \mathrm{~V}$. From the plasma floating value at the nozzle exit obtained with the electrostatic probe under this operating condition $(=-30 \pm 3 \mathrm{~V})$, a plasma potential value of $\approx-22 \mathrm{~V}$ can be inferred, ${ }^{8}$ and hence a total voltage drop of $=133 \pm 5 \mathrm{~V}$ can be derived as the voltage breakdown value along the sheath separating the unperturbed plasma and the nozzle wall for $\dot{m}=0.32 \mathrm{~g} \mathrm{~s}^{-1}$. For the other investigated $\dot{m}$ values the $\phi_{N}$ value could be lowered up to values very close to the cathode voltage without evidence of any breakdown.

From Fig. 3(a), note that the electron current drained from the arc is relatively small $\approx 1 \mathrm{~A}$, notwithstanding the fact that the size of the nozzle is quite large. This fact reflects the existence of a very low electron density close to the nozzle wall. Also note the great difference between the intensities of the ion and electron collected currents, indicating that in this experimental conditions electron attachment is not significant. These features were common to all the $\dot{m}$ values investigated.

Concerning to the ion branches shown in Fig. 3(b), note that the current drained by the nozzle increases when $\dot{m}$ decreases. Also note that no ion saturation current was found. This behavior is similar to those presented in wall-stabilized arc experiments with ${ }^{12}$ or without externally imposed axial gas flow. ${ }^{13}$

In order to get some information from the experimentally determined breakdown at the lowest $\dot{m}$ value, we must mention that this breakdown will likely occur at the exit of the nozzle. ${ }^{9,14}$ In this case the nozzle voltage is very close to the cathode voltage [see the ion branch of Fig. 3(b) corresponding to the lowest $\dot{m}$ value], while the plasma voltage increases continuously from cathode to anode along the nozzle direction and the gas pressure decreases continuously along such direction. We will show later that the sheath thickness $(D)$ is not expected in this case to vary appreciably along the nozzle. Hence at the nozzle exit the voltage difference between the plasma and the nozzle is the largest and the gas pressure is the smallest, resulting in the more favorable conditions for a Townsend breakdown. This is the mechanism proposed by Nemchinsky ${ }^{14}$ to explain the doublearcing formation. According to Paschen's law ${ }^{15}$ the breakdown voltage value depends on $D$ times the gas density $n$. We have estimated the $n$ value by taking an outer value of $0.1 \mathrm{MPa}$ for the gas pressure, and by assuming a gas temperature $T_{h}$ close to the estimated nozzle inner wall temperature. For instance, in a similar torch but with a cooled nozzle, ${ }^{3}$ the used inner wall temperature was $700 \mathrm{~K}$ so we have chosen $T_{h} \approx 1000 \mathrm{~K}$. Using the inferred $n$ value and the total voltage drop $(133 \mathrm{~V})$ along the sheath thickness together with the experimentally determined Paschen's law for an oxygen $\operatorname{gas}^{16}$ a value $D=20 \pm 2 \mu \mathrm{m}$ was finally determined for the sheath thickness at the nozzle exit.

To relate $D$ with the plasma characteristic quantities one has to take into account that when the nozzle is biased to a voltage different from that corresponding to the plasma potential an electrical boundary layer appears between the unperturbed plasma and the nozzle wall, extending mostly in the radial direction for the geometry of the experiment. Due to the high operating pressure values and the relatively low electron density $\left(n_{e}\right)$ at the plasma boundary, we will assume that this layer is collisional. When the sheath is collisional and without ionization (because both the electron density and the thickness of the sheath are small), Blank ${ }^{17}$ and Franklin $^{18-20}$ showed that there is a smooth joining between the plasma and the space charge layer without the need of introducing a transitional sheath. Sheridan and Goree ${ }^{21}$ using a numerical two-fluid model showed for a collisional sheath that $D$ can be approximated in terms of the plasma-wall voltage drop $(\Delta \phi)$ as

$$
D \approx\left(5^{3 / 5} 2^{2 / 5} 3^{-1} u_{0}^{-2 / 5} \alpha^{-1 / 5} \eta^{3 / 5}\right) \lambda_{D s},
$$

where $u_{0} \equiv\left(v_{+s} / v_{B}\right)$ is the nondimensional ion velocity at the sheath entrance, $v_{B} \equiv \sqrt{(k / M) T_{e}}$ is the Bohm velocity ( $k$ is the Boltzmann constant, $M$ is the ion mass, and $T_{e}$ is the electron temperature), $\alpha \equiv\left(\lambda_{D s} / \lambda_{+}\right)$is a parameter that measures the number of ion collisions within the sheath $\left(\lambda_{D s}\right.$ is the electron Debye length at the sheath edge and $\lambda_{+}$ $\equiv\left(1 / \sigma_{0} n\right)$ is the collisional ion mean free path, with $\sigma_{0} \approx 5$ $\times 10^{-19} \mathrm{~m}^{2}$ the elastic cross section ${ }^{22}$ ), and $\eta \equiv$ $-\left(e \Delta \phi / k T_{e}\right)$ is the nondimensional plasma-wall voltage drop where $e$ is the electron charge. In our case $\Delta \phi \equiv \phi_{N}-\phi_{p}$ where $\phi_{p}$ is the plasma potential.

Franklin $^{23}$ showed that the ion speed at the entry of the sheath is given approximately by 


$$
v_{+s} \approx v_{B}\left(\frac{\lambda_{+}}{\lambda_{D s}}\right)^{1 / 2}
$$

replacement of Eq. (2) into Eq. (1) leads to a more compact expression for $D$ :

$$
D \approx 5^{3 / 5} 2^{2 / 5} 3^{-1} \eta^{3 / 5} \lambda_{D s}=2.2 \times 10^{4} \Delta \phi^{3 / 5} n_{e}^{-1 / 2} T_{e}^{-1 / 10} .
$$

in the last numerical equality of Eq. (3) all the physical variables are given in MKS units $\left(n_{e}\right.$ is the electron density). Equation (3) can be employed to obtain plasma quantity values if the values of $D$ and $\Delta \phi$ are known. This can be done at the nozzle exit, where both values were estimated above for the breakdown situation. To obtain a relation between $n_{e}$ and $T_{e}$ we will consider that although the plasma close to the nozzle wall region is not in LTE equilibrium, the particle population in the different energy levels is still dominated by electron collisions. In this case, the electron temperature will result very different (and higher) than the heavy particle (ions and neutrals) temperature, and the nonequilibrium generalized Saha equation derived by van de Sanden et al. ${ }^{24}$ is appropriate to describe the plasma composition. In fact, this generalized Saha equation that employs the electron temperature instead of a unique ("thermodynamic") temperature, has been used in a numerical code for cutting torches. ${ }^{11}$ For the first ionization,

$$
\frac{n_{e} n_{i}}{n}=2 \frac{Q_{i}}{Q_{0}}\left(\frac{2 \pi m k T_{e}}{h^{2}}\right)^{3 / 2} \exp \left(-\frac{E_{I}-\delta E_{I}}{k T_{e}}\right),
$$

where $n_{i}$ is the ion density, $Q_{i}$ and $Q_{o}$ are the statistical weights of oxygen ions and atoms, $m$ is the electron mass and $E_{I}-\delta E_{I}$ is the ionization energy corrected by density effects (resulting in a negligible value $\delta E_{I} \approx 0.01 \mathrm{eV}$ for this case $\left.^{10}\right)$.

Also a neutrality equation can be written for the unperturbed plasma region at the sheath edge,

$$
n_{i} \cong n_{e} .
$$

Since at the nozzle exit the gas pressure $p \approx 0.1 \mathrm{MPa}$, the neutral gas density can be immediately obtained from the equation of state with the assumed value of the neutral temperature $(1000 \mathrm{~K})$ and neglecting the plasma pressure, resulting in $n \approx 7.2 \times 10^{24} \mathrm{~m}^{-3}$. So, replacing Eq. (5) into Eq. (4), one obtains a $2 \times 2$ system of equations [Eqs. (3) and (4)] to solve for $n_{e}$ and $T_{e}$. The resulting values at the nozzle exit where $n_{e} \approx 7.5 \times 10^{19} \mathrm{~m}^{-3}$ and $T_{e} \approx 5700 \mathrm{~K}$. Note that the quoted value of $n_{e}$ is well below the lower limiting value to apply LTE equilibrium in accord with Griem's criterion.

The physical picture presented up until now can be used to look for an explanation of the nozzle ion branch characteristic. The ion current $\left(i_{+}\right)$collected by the nozzle can be written as

$$
i_{+}=2 \pi R_{N} e \int_{z=0}^{z=L_{N}} n_{i} v_{+s} d z
$$

where $z$ is the coordinate directed along the nozzle wall and the ion density and velocity must be evaluated at the sheath edge. According to the set of equations previously presented Eqs. (1)-(5), the ion current collected by the nozzle depends on the values of several quantities of the unperturbed plasma-gas structure at the sheath edge $\left(n, n_{e}\right.$, and $\left.T_{e}\right)$. Since the sheath thickness depends on the nozzle voltage value [Eq. (3)], an appropriate way within the presented physical frame to explain the observed behavior of the ion branch characteristic is to assume relevant radial variations of these quantities. This means that a larger $\Delta \phi$ value produces a thicker sheath and hence the plasma is "probed" at different radial positions producing different current values to the nozzle. In fact, the radial position values at which the plasma quantities will be evaluated are given by Eq. (3) for each value of $\phi_{N}$ It is interesting to note that, since the expected values of $T_{e}$ are around 5000-6000 K (according to what was found with the breakdown estimation), the generalized Saha Eq. (3) predicts a very strong dependence of $n_{e}$ on $T_{e}$, thus resulting in very small variations of $T_{e}$ that are able to produce large enough variations of $n_{e}$ that, in turn, can explain the behavior of the characteristic.

In order to invert Eq. (6), we will assume a linear variation of $p$ and $\phi_{p}$ with $z$, in accord with what is shown by several numerical simulations. ${ }^{6,11}$ Note that the equation of state states a relation between $p$ and $n$, since the plasma pressure is negligible. In any case, the obtained solution is almost insensitive to the particular chosen variations of $p$, since the dependence of the integral in Eq. (6) with $n$ is very weak, leading to a dependence of the kind $n^{1 / 8}$. To calculate the electrostatic potential of the plasma it is necessary to know its value in two different axial positions. One of them, at the nozzle exit, has been previously inferred from the experimentally determined plasma floating potential. The second point can be taken at the nozzle entrance, since at that position the plasma potential will be very close to the nozzle floating potential, following arguments clearly presented by Nemchinsky and Severance ${ }^{9}$ in a recent review. For instance, for $\dot{m}=0.32 \mathrm{~g} \mathrm{~s}^{-1}$ the values of $\phi_{p}$ are $\phi_{p}\left(z=L_{N}\right)=-22 \mathrm{~V}$ and $\phi_{p}(z=0)=-70 \mathrm{~V}$ [see Fig. 3(b)]. The resulting value of the constant axial electric field in this case is $11 \mathrm{~V} / \mathrm{mm}$.

With the above quoted assumptions, the neutral gas density is a known function of $z$, so to solve Eq. (6) (that gives the collected current integrated along the nozzle length) it is necessary to know the axial dependences of $n_{e}$ and $T_{e}$. Actually, the generalized Saha equation relates both variables, so it is necessary to know the axial dependence of only one of them. Since there is no clear criterion to perform any choice for the quoted dependence, and guided by the published solution of a numerical code for cutting torches, ${ }^{11}$ which shows remarkable constant values of the electron temperature along the nozzle close to the wall, we have chosen the simplest assumption of considering a constant value (an average along the nozzle length) for $T_{e}$ [so $T_{e}(r)$, where $r$ is the radial coordinate]. Therefore, $T_{e}$ only depends on the radial coordinate $r$ and we can write $T_{e}$ as a polynomial expansion

$$
T_{e}(r)=a_{0}+a_{1} r+a_{2} r^{2}+\cdots+a_{q-1} r^{q-1},
$$

where $a_{0}, a_{1}, \ldots, a_{q-1}$ are $q$ unknown constant numerical coefficients. Then replacing $n_{e}$ in terms of $T_{e}$ [evaluated at the sheath edge $\left.T_{e}\left(r=R_{N}-D\right)\right]$ through Saha equation in Eq. (3), we can found the $z$ dependence of the sheath thickness $D$ as 


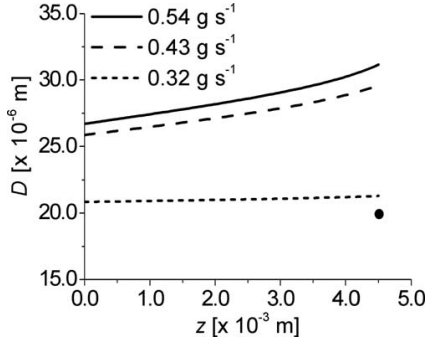

FIG. 4. Sheath thickness along the nozzle wall for the largest nozzle voltage value experimentally registered for each gas mass flow value. For comparative purposes, the black circle indicates the sheath thickness value inferred at the nozzle exit using the voltage breakdown measurement.

a function of the quoted unknown numerical coefficients.

Using Eq. (7) evaluated at $T_{e}\left[r=R_{N}-D(z)\right]$, and expressing $n_{e}$ as a function of $T_{e}$, the axial dependence of the integrand in Eq. (6) is known in terms of $\phi_{N}$ and the unknown $q$ numerical coefficients. Hence Eq. (6) can be integrated for $q$ points of the ion branch characteristic $\left(i_{+}^{(j)}-\phi_{N}^{(j)}\right)$ to produce a $q \times q$ system of equations

$$
\begin{aligned}
i_{+}^{(j)} & =2 \pi R_{N} e \int_{z=0}^{z=L_{N}} \psi\left(\phi_{N}^{(j)}, a_{0}, a_{1}, \ldots, a_{q-1}, z\right) d z,(j \\
& =1,2,3, \ldots, q) .
\end{aligned}
$$

For reasons that will be explained later, only the $\phi_{N}$ experimental values sufficiently far from the characteristic floating potential have been included in Eq. (8).

With all these considerations, and using the well-known Chebyshev formula ${ }^{25}$ to approximate the integrals of this system of equations, the inversion problem finally can be solved calculating the solution of the $q \times q$ system of equations given by Eq. (8), in terms of the $q$ numerical coefficients in Eq. (7). Thus we obtain the $T_{e}(r)$ profile that fits the characteristic ion branch, in the radial range of the $D$ values.

In practice for all the studied cases, a linear variation of $T_{e}(r)$ was found sufficient to fit the characteristic ion branches within the experimental uncertainty $( \pm 5 \%)$, which results from statistical fluctuations of the arc current and voltage.

In Fig. $4 D(z)$ for the different $\dot{m}$ values and for the largest nozzle voltage value registered in each characteristic ion branch is presented. It can be seen that for the lowest gas mass flow value $\left(\dot{m}=0.32 \mathrm{~g} \mathrm{~s}^{-1}\right)$ an almost constant $D(z)$ $\approx 21 \mu \mathrm{m}$ results. Note that the sheath thickness at the nozzle exit value $\left(D\left(z=L_{N}\right)=20 \mu \mathrm{m}\right)$ inferred from the voltage breakdown measurement for this case is quite close to the predicted value. In the other cases, a more marked increase with $z$ appears in $D(z)$, varying in the range 26-30 $\mu \mathrm{m}$ for $\dot{m}=0.43 \mathrm{~g} \mathrm{~s}^{-1}$ and $27-31 \mu \mathrm{m}$ for $\dot{m}=0.54 \mathrm{~g} \mathrm{~s}^{-1}$. These values are higher than the sheath thickness value corresponding to the lowest gas mass flow case, which is consistent with the lack of breakdown found in these cases. Concerning the validity of the collisional sheath assumption, the ratio of the sheath thickness to the ion mean free path $\lambda_{+}$results $D / \lambda_{+}$ $\approx 100$, showing that the above-mentioned assumption is fully satisfied.

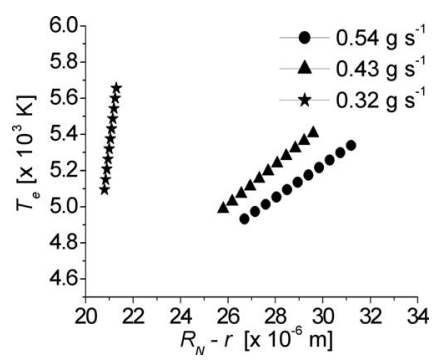

FIG. 5. Radial profiles of the average (along the nozzle wall) plasma electron temperature as a function of the radial position measured from the nozzle wall, for the different gas mass flow values.

In Fig. 5 the radial profiles of $T_{e}$ corresponding to the registered ion branch characteristics are presented as a function of the radial coordinate measured from the nozzle wall $\left(R_{N}-r\right)$. Figure 5 shows that the values of $T_{e}$ for the different $\dot{m}$ values are not very different (4700-5700 K), but there appear large and quite different $T_{e}$ radial gradients. In fact, gradients of the order of $0.9-1.1 \times 10^{8} \mathrm{~K} \mathrm{~m}^{-1}$ for $\dot{m}$ $=0.43-0.54 \mathrm{~g} \mathrm{~s}^{-1}$ and $1.1 \times 10^{9} \mathrm{~K} \mathrm{~m}^{-1}$ for $\dot{m}=0.32 \mathrm{~g} \mathrm{~s}^{-1}$ are found very close to the nozzle wall, thus supporting the assumption of the lack of LTE equilibrium.

The spatial distribution of the plasma density is presented in Figs. 6(a) and 6(b), for $\dot{m}=0.32$ and $0.54 \mathrm{~g} \mathrm{~s}^{-1}$, respectively. A steep plasma density radial gradient is shown in Fig. 6(a) due to the existence of very large electron temperature radial gradient close to the nozzle wall (see Fig. 5). Less marked plasma density gradients close to the nozzle wall are shown in Fig. 6(b) for the largest gas mass flow rate registered. In both figures the existence of a plasma pressure gradient along the nozzle produces the axial variation in the plasma density.

Guided for the experimental and theoretical results given by Figs. 3-6, we can now discuss the physical origin of the double-arcing phenomenon. In practice, double arcing is produced when the gas mass flow is too low, ${ }^{9,14}$ which is seen to occur in our case. The experimental results varying the $\dot{m}$
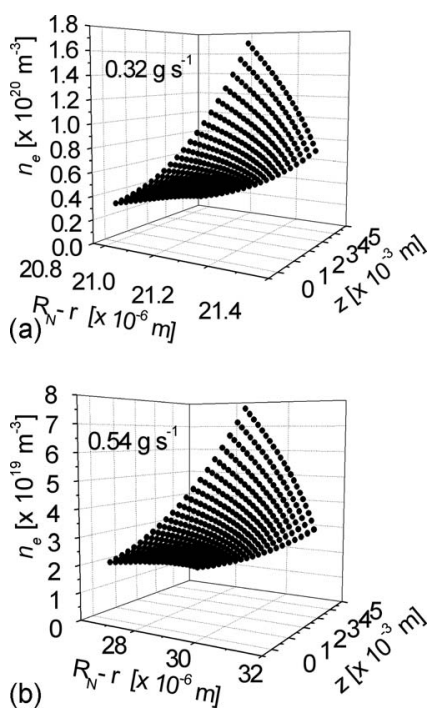

FIG. 6. (a) Spatial distribution of the plasma density close to the nozzle wall for the lowest gas mass flow experimentally registered. (b) Spatial distribution of the plasma density close to the nozzle wall for the largest gas mass flow experimentally registered. 
value show that the current drained by the nozzle increases [see Fig. 3(b)] when the gas mass flow decreases until finally a breakdown appears. According to our interpretation, the physical structure of the plasma boundary layer is strongly influenced by the gas mass flow rate (see Figs. 4-6), in the sense that the $T_{e}$ profile critically depends on the $\dot{m}$ value. When the $\dot{m}$ value decreases, very large $T_{e}$ radial gradients (of the order of $10^{9} \mathrm{~K} \mathrm{~m}^{-1}$ ) produce (through Saha equation) steep $n_{e}$ radial gradients over the last few Debye electron lengths from the nozzle wall. As a result, the sheath thickness is almost independent of the $\Delta \phi$ value, producing an almost constant (and thin) sheath thickness along the nozzle for low $\dot{m}$ values. Once the sheath thickness is smaller than the critical value, the cold gas envelope breaks down leading to double arcing. A good agreement between the critical value of the sheath thickness at the nozzle exit inferred from the voltage breakdown (under the Townsend breakdown hypothesis) and the predicted value is found.

A possible photoelectron current from the nozzle inner surface deserves some analysis. It should be noted that in this kind of cutting torches, the arc is extremely constricted, resulting in high pressures, high current densities, and correspondingly high temperatures on the arc axis. So the radiative arc loses are a significant fraction of the electrical power input, and it is found that the spectral contribution at wavelengths less than $200 \mathrm{~nm}$ (photon energies of about $6 \mathrm{eV}$ ) is important in a wide range of conditions. ${ }^{26}$ Using the semiempirical radiative model of Zhang et al. ${ }^{27}$ and a copper photoemission coefficient $\gamma_{\nu} \approx 10^{-4}$ (Ref. 28) we have found that the photoemission from the nozzle surface yields a maximum electron current of $\approx 2.5 \mathrm{~mA}$. This photoelectron current will not dependent on the nozzle voltage, and only could produce a change in the characteristic curves at the vicinities of the nozzle floating voltage value [see Fig. 3(b)]. For this reason, the characteristic region near the floating voltage was not considered in the inversion of Eq. (6).

\section{FINAL REMARKS}

In this work we have presented a diagnostic for a cutting arc, consisting in obtaining the nozzle current-voltage characteristic. The measurements are reported using a high energy density cutting arc torch with a nozzle bore radius of 0.5 $\mathrm{mm}$. The arc current was fixed at $30 \mathrm{~A}$ while the plenum pressure and the oxygen gas mass flow rate were varied in the ranges of $0.55-0.65 \mathrm{MPa}$ and $0.32-0.54 \mathrm{~g} \mathrm{~s}^{-1}$, respectively. The results showed a very low electron density and the lack of electron attachment at the plasma boundary layer. No ion saturation current was found. For the smallest $\dot{m}$ value it was found a breakdown for a biasing nozzle potential close to that of the cathode, but no evidence of such breakdown was found for the larger $\dot{m}$ values.

Using an expression for the ion speed at the entry of the collisional sheath formed between the nonequilibrium plasma and the negatively biased nozzle wall ${ }^{23}$ together with a generalized Saha equation ${ }^{24}$ coupled to the ion branch of the characteristic, the radial profile of the electron temperature, the spatial distribution of the plasma density at the plasma boundary, and the sheath thickness are obtained. In particular, the obtained thickness value at the breakdown condition is in good agreement with that obtained from the oxygen Paschen's curve. An electron temperature of about $4700-5700 \mathrm{~K}$ and a corresponding plasma density of the order of $10^{19}-10^{20} \mathrm{~m}^{-3}$ were found close to the nozzle wall. Very high temperature gradients (of the order of $10^{8}-10^{9} \mathrm{~K} \mathrm{~m}^{-1}$, depending on the $\dot{m}$ value) over the last few electron Debye lengths from the nozzle wall are predicted.

The obtained results have allowed to discuss the physical origin of the double-arcing phenomenon that appears experimentally at low $\dot{m}$ values. In this case, an almost constant (and thin) sheath thickness along the nozzle is obtained. Once the sheath thickness is smaller than the critical value (predicted by Paschen's law), the cold envelope breaks down leading to double arcing.

\section{ACKNOWLEDGMENTS}

This work was supported by grants from the Universidad de Buenos Aires (No. PID X111), CONICET (No. PIP 5378), and UTN (No. PID Z 0007). One of the authors (L.P.) is grateful to the Fundación YPF. Also B.M. is grateful to the Universidad Tecnológica Nacional. H.K. is a member of the CONICET.

${ }^{1}$ S. Ramakrishnan, M. Gershenzon, F. Polivka, T. N. Kearny, and M. W. Rogozinsky, IEEE Trans. Plasma Sci. 25, 937 (1997).

${ }^{2}$ L. Girard, Ph. Teulet, M. Razafinimanana, A. Gleizes, F. Camy-Peyret, E. Baillot, and F. Richard, J. Phys. D: Appl. Phys. 39, 1543 (2006).

${ }^{3}$ P. Freton, J. J. Gonzalez, A. Gleizes, F. Camy Peyret, G. Caillibotte, and M. Delzenne, J. Phys. D: Appl. Phys. 35, 115 (2002).

${ }^{4}$ P. Freton, J. J. Gonzalez, A. Gleizes, F. Camy Peyret, G. Caillibotte, and M. Delzenne, J. Phys. D: Appl. Phys. 35, 115 (2002).

${ }^{5}$ C. Pardo, J. González-Aguilar, A. Rodríguez Yunta, and M. A. G. Calderón, J. Phys. D: Appl. Phys. 32, 2181 (1999).

${ }^{6}$ J. González-Aguilar, C. Pardo Sanjurjo, A. Rodríguez Yunta, and M. A. García Calderón, IEEE Trans. Plasma Sci. 27, 264 (1999).

${ }^{7}$ L. Prevosto, H. Kelly, and B. Mancinelli, IEEE Trans. Plasma Sci. 36, 263 (2008).

${ }^{8}$ L. Prevosto, H. Kelly, and B. Mancinelli, IEEE Trans. Plasma Sci. 36, 271 (2008).

${ }^{9}$ V. A. Nemchinsky and W. S. Severance, J. Phys. D: Appl. Phys. 39, R423 (2006).

${ }^{10}$ M. Boulos, P. Fauchais, and E. Pfender, Thermal Plasmas, Fundamentals and Applications (Plenum, New York, 1994).

${ }^{11}$ S. Ghorui, J. V. R. Heberlein, and P. E. Pfender, J. Phys. D: Appl. Phys. 40, 1966 (2007).

${ }^{12}$ R. J. Hill and G. R. Jones, J. Phys. D: Appl. Phys. 12, 1707 (1979).

${ }^{13}$ D. W. George and P. H. Richards, Br. J. Appl. Phys. 1, 1171 (1968).

${ }^{14}$ V. A. Nemchinsky, J. Phys. D: Appl. Phys. 31, 3102 (1998).

${ }^{15}$ P. Raizer, Gas Discharge Physics (Springer, Berlin, 1991).

${ }^{16}$ R. Hackam, J. Phys. B 2, 216 (1969).

${ }^{17}$ J. L. Blank, Phys. Fluids 11, 1686 (1968).

${ }^{18}$ R. N. Franklin, J. Phys. D: Appl. Phys. 37, 1342 (2004).

${ }^{19}$ R. N. Franklin, J. Phys. D: Appl. Phys. 36, R309 (2003).

${ }^{20}$ R. N. Franklin, J. Phys. D: Appl. Phys. 36, 2821 (2003).

${ }^{21}$ T. E. Sheridan and J. Goree, Phys. Fluids B 3, 2796 (1991).

${ }^{22}$ H. L. Anderson, A Physicist's Desk Reference, 2nd ed. (AIP, New York, 1989).

${ }^{23}$ R. N. Franklin, IEEE Trans. Plasma Sci. 30, 352 (2002).

${ }^{24}$ M. C. M. van de Sanden, P. P. J. M. Schram, A. G. Peeters, J. A. M. van der Mullen, and G. M. W. Kroesen, Phys. Rev. A 40, 5273 (1989).

${ }^{25}$ B. Noble, Numerical Methods: 2 Differences, Integration and Differential Equations (Oliver and Boyd, Edinburgh, 1964).

${ }^{26}$ P. J. Shayler and M. T. C. Fang, J. Phys. D: Appl. Phys. 11, 1743 (1978).

${ }^{27}$ J. F. Zhang, M. T. C. Fang, and D. B. Newland, J. Phys. D: Appl. Phys. 20, 368 (1987)

${ }^{28}$ D. H. Dowell, F. K. King, R. E. Kirby, and J. F. Schemerge, Phys. Rev. ST Accel. Beams 9, 063502 (2006). 